\title{
Revista Colombiana de

\section{Reporte de un caso de ruptura del músculo papilar anterior debido a infarto agudo de miocardio}

\author{
César D. Niño-Pulido ${ }^{a}$, Ana G. Múnera-Echeverri ${ }^{\mathrm{b}, *}$, César A. Hernández ${ }^{\mathrm{a}, \mathrm{b}, \mathrm{c}}$, \\ Jorge A. Arroyave ${ }^{\mathrm{a}, \mathrm{b}}$, José L. Ramírez ${ }^{\mathrm{a}, \mathrm{b}, \mathrm{d}}$ y Mauricio Duque-Ramírez ${ }^{\mathrm{a}}$
}

\author{
a Universidad CES, CES Cardiología, Medellín, Colombia \\ ${ }^{\mathrm{b}}$ Hospital General de Medellín Luz Castro de Gutiérrez, Medellín, Colombia \\ c Clínica Las Vegas, Medellín, Colombia \\ d Universidad Pontifica Bolivariana, Medellín, Colombia
}

Recibido el 30 de mayo de 2017; aceptado el 1 de marzo de 2018

Disponible en Internet el 7 de junio de 2018

\section{PALABRAS CLAVE Infarto agudo de miocardio; Insuficiencia mitral; Cambio valvular; Cirugía; Ecocardiografía}

\section{KEYWORDS}

Acute myocardial infarction;

Mitral insufficiency;

Valve replacement;

Surgery;

Echocardiography

\begin{abstract}
Resumen La ruptura del músculo papilar anterolateral es una complicación del síndrome coronario agudo poco frecuente, en especial porque este músculo usualmente tiene doble irrigación sanguínea al comparar con el músculo papilar posteromedial que, por lo general, solo tiene una irrigación. Se presenta el caso de un paciente que consultó por signos y síntomas de falla cardiaca aguda precedidos de dolor de pecho, en quien se documentó insuficiencia mitral grave por ruptura del músculo papilar anterolateral debido a enfermedad coronaria isquémica de múltiples vasos.

(C) 2018 Sociedad Colombiana de Cardiología y Cirugía Cardiovascular. Publicado por Elsevier España, S.L.U. Este es un artículo Open Access bajo la licencia CC BY-NC-ND (http:// creativecommons.org/licenses/by-nc-nd/4.0/).
\end{abstract}

Anterior papillary muscle rupture due to an acute myocardial infarction: A case
report

\begin{abstract}
The rupture of the anterior-lateral papillary muscle is an uncommon complication of acute coronary syndrome. This is particularly relevant as this has double blood irrigation compared to that of the posteromedial papillary muscle that generally only has single irrigation. The case is presented on a patient that consulted due to having signs and symptoms of acute heart failure preceded by chest pains. Severe mitral insufficiency was observed due to a rupture of the anterior-lateral papillary muscle caused by a multiple vessel ischaemic coronary disease. (c) 2018 Sociedad Colombiana de Cardiología y Cirugía Cardiovascular. Published by Elsevier España, S.L.U. This is an open access article under the CC BY-NC-ND license (http:// creativecommons.org/licenses/by-nc-nd/4.0/).
\end{abstract}

\footnotetext{
* Autor para correspondencia.

Correo electrónico: anagm@une.net.co (A.G. Múnera-Echeverri).
} 


\section{Introducción}

La ruptura de un músculo papilar es una complicación mecánica muy grave de la enfermedad coronaria isquémica ${ }^{1}$. Esta ocurría principalmente en el síndrome coronario agudo con elevación del ST, pero su incidencia ha disminuido y ha aumentado en síndrome coronario agudo sin elevación del ST, cambio que puede explicarse por disminución en la incidencia de síndrome coronario agudo con elevación del ST y aumento en la incidencia de síndrome coronario agudo sin elevación del ST, presentación clínica más indolente en el síndrome coronario agudo sin elevación del ST que lleva a diagnóstico y manejo tardío; y revascularización temprana en el síndrome coronario agudo con elevación del ST que reduce la incidencia de complicaciones mecánicas ${ }^{2}$. Es así como el intervencionismo percutáneo ha disminuido la incidencia de estas complicaciones a menos del 1\%; la ruptura del músculo papilar ocurre en un $0,26 \%$ de los casos, la de la pared libre en un $0,52 \%$ y la del septum ventricular en un $0,17 \%{ }^{1}$. En caso de presentarse ruptura del músculo papilar la mortalidad puede ser hasta del $80 \%$ durante la primera semana del infarto ${ }^{3}$ y explica hasta el $7 \%$ de los casos de choque cardiogénico por infarto agudo de miocardio ${ }^{4}$. La ruptura del músculo papilar posteromedial es más común (75\% de los casos) porque generalmente se irriga de un único vaso (descendente posterior) y se asocia a infarto de pared inferior. La ruptura del músculo anterolateral es menos común ( $25 \%$ de los casos), porque éste usualmente tiene doble irrigación (obtusa marginal de la arteria circunfleja y primera diagonal de la arteria descendente anterior); se asocia a infarto de pared anterior ${ }^{5}$. En un estudio se encontró variación en la irrigación de estos músculos; el músculo papilar anterior tenía doble irrigación en $71 \%$ de los pacientes, en comparación con $37 \%$ en el músculo papilar posterior. Cuando hay doble irrigación es menos probable la ruptura ${ }^{6}$.

\section{Caso}

Paciente de 61 años con antecedente personal de hipertensión en tratamiento con losartán e hidroclorotiazida, exfumador de 3 paquetes/año e historia familiar de muerte por infarto (madre a los 52 años). Consultó al servicio de urgencias del Hospital General de Medellín (Luz Castro de Gutiérrez) por presentar cuadro clínico de dos meses de evolución que consistía en dolor de pecho opresivo no irradiado asociado a disnea con actividad física que mejoraba en reposo, con empeoramiento de los síntomas en reposo durante los últimos dos días, asociado a diaforesis, ortopnea, bendopnea y edema de miembros inferiores; durante la admisión tuvo mayor deterioro respiratorio y tendencia a la hipotensión.

Al examen físico se encontró polipneico con intolerancia al decúbito, presión arterial de $170 / 60 \mathrm{~mm} \mathrm{Hg}$ (luego 86/46), frecuencia cardiaca de 108 latidos por minuto, frecuencia respiratoria de 24 respiraciones por minuto, temperatura de $37^{\circ} \mathrm{C}$ y saturación de oxígeno de $82 \%$ al ambiente. Se observó dilatación de venas yugulares, ruidos cardiacos taquicárdicos con soplo sistólico mitral y S3, murmullo vesicular disminuido con crépitos en ambos campos pulmonares y edema grado II en miembros inferiores. El electrocardiograma inicial mostró ritmo sinusal con trastornos inespecíficos de la repolarización (sin elevación del ST) y en radiografía de tórax se apreciaban infiltrados intersticiales bilaterales y cardiomegalia (fig. 1).

Se trasladó a la unidad de cuidados especiales con diagnóstico de infarto agudo de miocardio sin elevación del ST (troponina T 2,27, valor de referencia $<0,012 \mathrm{ng} / \mathrm{ml}$ ), clasificación Killip III (luego Killip IV) en donde se trató de acuerdo con las guías vigentes ${ }^{7}$. Se le realizó una ecocardiografía transtorácica (fig. 2) que demostró ventrículo izquierdo con remodelamiento concéntrico, hipocinesia basal de la pared inferior e hipercontractilidad de los demás segmentos, fracción de eyección del 70\%, disfunción diastólica, ventrículo derecho normal con presión sistólica de la pulmonar calculada de $50 \mathrm{~mm} \mathrm{Hg}$, leve dilatación de la aurícula izquierda e insuficiencia severa excéntrica anterior de la válvula mitral con imágenes de prolapso del músculo papilar anterolateral en el segmento P2. Estos hallazgos se corroboraron con ecografía transesofágica (fig. 2). Con lo anterior, se llevó a coronariografía diagnóstica (fig. 2) en la que se evidenció lesión estenótica del $60 \%$ de la arteria descendente anterior en la bifurcación de la segunda diagonal y en porción distal del $80 \%$; primera y segunda diagonal con lesión del $70 \%$ proximal, arteria circunfleja con ostium independiente y lesión en tercio medio a nivel de bifurcación de la tercera obtusa marginal del 93\%, primera obtusa marginal con lesión del 99\% con flujo TIMI II, arteria coronaria derecha dominante con lesiones difusas no significativas, arteria descendente posterior y arteria posterolateral con lesiones del $50 \%$ a nivel de la bifurcación. Se dejó con balón de contrapulsación aórtica 1:1.

Fue llevado a cirugía para remplazo valvular (bioprótesis Mosaic número 27) y revascularización quirúrgica de tres puentes con arteria mamaria interna izquierda a arteria descendente anterior, safena a primera obtusa marginal y safena a primera diagonal (fig. 3); requirió reintervención por sangrado persistente, luego de lo cual evolucionó favorablemente y fue dado de alta.

\section{Discusión}

Se presenta el caso de un paciente con una complicación mecánica de infarto agudo de miocardio con enfermedad coronaria de tres vasos, con vaso culpable la primera obtusa marginal e insuficiencia mitral severa debido a ruptura del músculo papilar anterior, lo cual se evidenció desde la ecografía transtorácica y se confirmó mediante ecografía transesofágica.

La válvula mitral tiene dos músculos papilares cuya función es evitar el prolapso de la valva anterior con el músculo posteromedial y la valva posterior con el músculo anterolateral. La ruptura de un músculo papilar lleva a insuficiencia mitral severa aguda seguida de falla del ventrículo izquierdo, choque cardiogénico y muerte ${ }^{1,8}$. La causa más frecuente de ruptura o disfunción de los músculos papilares es la isquémica9 ${ }^{9}$, si bien antes lo era en síndrome coronario agudo con elevación del ST; pero, gracias al intervencionismo percutáneo y al aumento en la incidencia de síndrome coronario agudo sin elevación del ST, es más frecuente en síndrome coronario sin elevación del $\mathrm{ST}^{2}$.

Se requiere un alto índice de sospecha basado en la historia clínica, el examen físico (un soplo sistólico mitral) y en la evaluación ecocardiográfica temprana en los pacientes inestables ${ }^{1,3}$. 

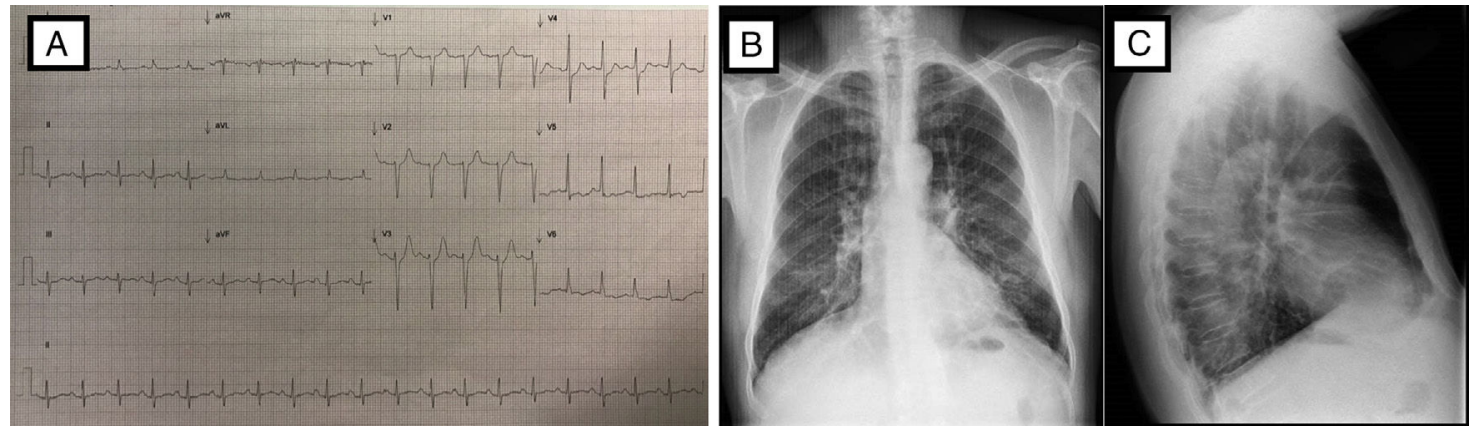

Figura 1 Estudios al Ingreso. A) Electrocardiograma: Taquicardia sinusal, eje $-10^{\circ} \mathrm{C}, \mathrm{rS}$ en DIII, aVF, pobre progresión $\mathrm{r}$ de V1-V3, Duración de PR, QRS y QTc normal, no elevación ST, depresión del ST de V4-V6. B y C) Radiografía de tórax PA y lateral: índice cardiotorácico normal, leve reforzamiento vascular parahiliar bilateral, infiltrado intersticial escaso bilateral y escaso derrame izquierdo posterior.
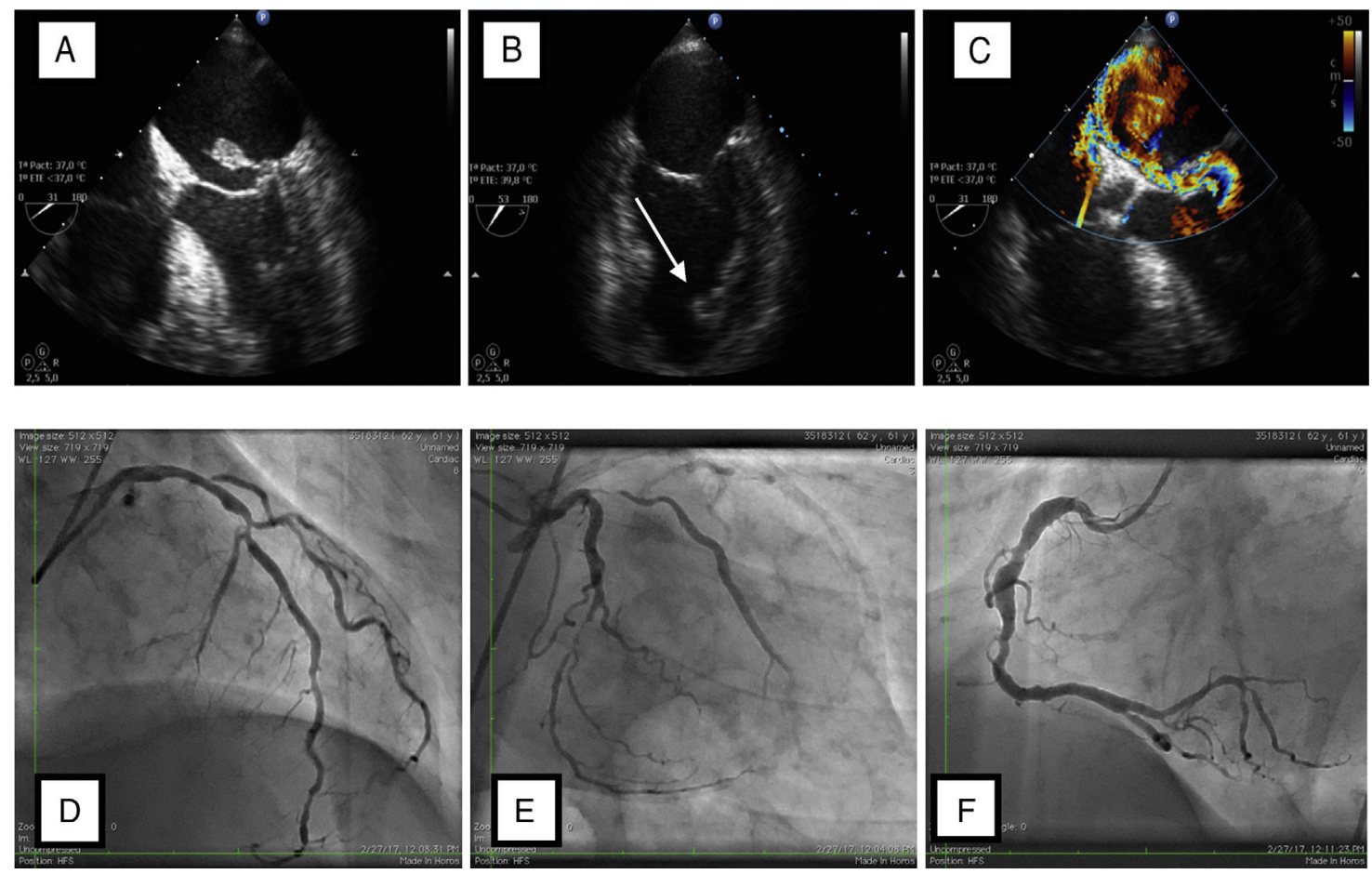

Figura 2 Ecocardiografía A) Ecocardiografía transesofágica, eje a $31^{\circ}$, prolapso de cabeza músculo papilar con cuerdas tendinosas en la aurícula izquierda. B) Ecocardiografía transesofágica, eje $53^{\circ}$, dos cámaras ; se aprecia músculo papilar anterior amputado (flecha blanca). C) Ecocardiografía transesofágica con doppler color, eje $31^{\circ}$, insuficiencia mitral anterior severa. Coronariografía D) Proyección oblicua con craneal, lesión estenótica de $60 \%$ de la arteria descendente anterior a nivel de la bifurcación de la segunda diagonal y en porción distal del $80 \%$, la primera con lesión del $80 \%$ proximal y segunda diagonal con lesión del $70 \%$ proximal. E) Proyección $10^{\circ}$ derecho y caudal $30^{\circ}$, arteria circunfleja con ostium independiente, primera obtusa marginal con lesión del $99 \%$ y flujo TIMI II, circunfleja con lesión en tercio medio a nivel de bifurcación de la tercera obtusa marginal del 93\%. F) Proyección oblicua izquierda a 45 grados, arteria coronaria derecha dominante con lesiones difusas, arteria descendente posterior y arteria posterolateral con lesiones del $50 \%$ a nivel de la bifurcación.

El intervalo entre el establecimiento de los síntomas y la ruptura del músculo papilar varía entre 6 horas y 7 días ${ }^{10}$.

El diagnóstico y tratamiento oportunos mejoran el pronóstico ${ }^{4}$, apoyados en la historia clínica, los hallazgos al examen físico, el electrocardiograma y la ecocardiografía ${ }^{1,4}$. El manejo inicial debe ser médico enfocado en el infarto y en la reducción de la poscarga, para aumentar el volumen sistólico, incrementar así el gasto cardiaco y reducir el volumen regurgitante. Para lo anterior se usan vasodilatadores y balón de contrapulsación aórtica, y en algunos pacientes hasta dispositivos de soporte mecánico, membranas de oxigenación extracorpórea e hipotermia ${ }^{1,11}$.

Se han reportado buenos resultados tanto para el cambio valvular como para la reparación valvular, pero parece haber mejor resultado en pacientes que tienen reparación valvular cuando es posible $\mathrm{e}^{12-14}$. En otro estudio retrospectivo se analizaron pacientes durante los años 1980 a 2000 y se demostró una diferencia en mortalidad perioperatoria entre reparación $7,7 \%$ y cambio valvular $22 \%$ ( $p=0,21$, no significativo por bajo tamaño de muestra en el grupo de reparación); 

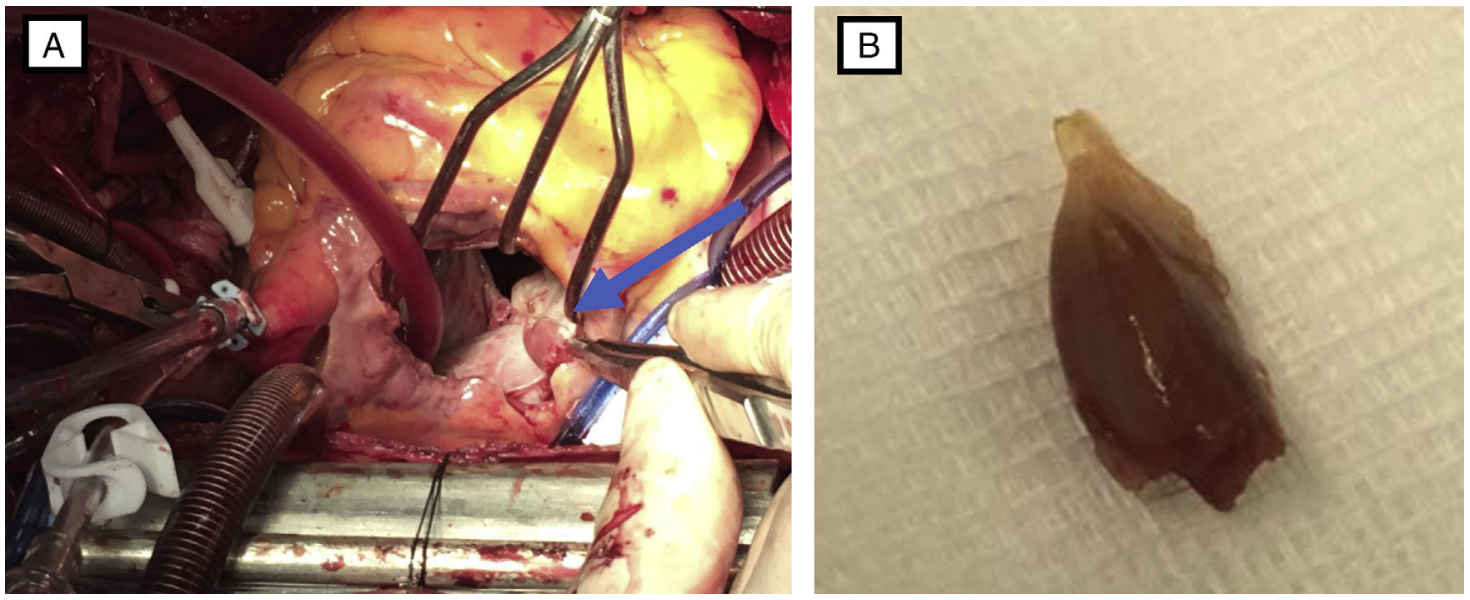

Figura 3 Pieza quirúrgica. A) Fotografía de ruptura de músculo papilar anterior (flecha azul). B) Fotografía de ruptura del músculo papilar extraído.

así mismo, se demostró menor mortalidad perioperatoria al hacer revascularización simultánea (OR 0,18;0,04 - 0,83, $\mathrm{p}=0,011)$ y tendencia a mayor supervivencia a largo plazo $(\text { OR } 0,45 ; 0,2-1,1, p=0,077)^{15}$.

Los hallazgos en el paciente del caso fueron similares a los de otros reportes de caso $^{3,16}$, donde el estado clínico, la historia clínica y el examen físico fueron claves para sospechar una complicación mecánica, confirmada con la ecocardiografía y tratada de manera oportuna con cirugía.

\section{Conclusión}

Las complicaciones mecánicas de los eventos isquémicos agudos afectan negativamente el pronóstico de los pacientes; es así como se recomienda una evaluación ecocardiográfica inmediata cuando se sospeche una complicación mecánica, en cuyo caso el manejo definitivo será la cirugía, la cual se hará tan pronto como sea posible.

\section{Financiación}

Ninguna.

\section{Conflicto de intereses}

Ninguno.

\section{Bibliografía}

1. Kutty RS, Jones N, Moorjani N. Mechanical complications of acute myocardial infarction. Cardiol Clin. 2013;31:519-31, viiviii.

2. Murad K, Missov E. Papillary muscle rupture following non-ST-elevation myocardial infarction: a case report. Echocardiography. 2016;33:923-5.

3. Hutyra M, Skala T, Marek D, Nemec P, Sluka M, Ostransky J, et al. Acute severe mitral regurgitation with cardiogenic shock caused by two-step complete anterior papillary muscle rupture during acute myocardial infarction. Biomed Pap Med Fac Univ Palacky Olomouc Czech Repub. 2006;150:293-7.

4. Thompson CR, Buller CE, Sleeper LA, Antonelli TA, Webb JG, Jaber WA, et al. Cardiogenic shock due to acute severe mitral regurgitation complicating acute myocardial infarction: a report from the SHOCK Trial Registry, SHould we use emergently revascularize Occluded Coronaries in cardiogenic shock? J Am Coll Cardiol. 2000;36 3 Suppl A:1104-9.

5. Wada H, Yasu T, Murata S, Ohta M, Kubo N, Fujii M, et al. Rupture of the anterolateral papillary muscle caused by a single diagonal branch obstruction. Circ J. 2002;66:872-3.

6. Voci P, Bilotta F, Caretta Q, Mercanti C, Marino B. Papillary muscle perfusion pattern. A hypothesis for ischemic papillary muscle dysfunction. Circulation. 1995;91:1714-8.

7. Roffi M, Patrono C, Collet JP, Mueller C, Valgimigli M, Andreotti F, et al. 2015 ESC Guidelines for the management of acute coronary syndromes in patients presenting without persistent ST-segment elevation: Task Force for the Management of Acute Coronary Syndromes in Patients Presenting without Persistent ST-Segment Elevation of the European Society of Cardiology (ESC). Eur Heart J. 2016;37:267-315.

8. Yamazaki M, Fukui T, Mahara K, Takanashi S. Complete rupture of the anterolateral papillary muscle caused by coronary spasm. Interact Cardiovasc Thorac Surg. 2015;21:798-800.

9. Roberts WC, Ko JM. Some observations on mitral and aortic valve disease. Proc (Bayl Univ Med Cent). 2008;21:282-99.

10. Nishimura RA, Schaff HV, Shub C, Gersh BJ, Edwards WD, Tajik AJ. Papillary muscle rupture complicating acute myocardial infarction: analysis of 17 patients. Am J Cardiol. 1983;51:373-7.

11. Güvenç RÇ, Güvenç TS. Clinical presentation, diagnosis and management of acute mitral regurgitation following acute myocardial infarction. J Acute Dis. 2016;5:96-101.

12. Rankin JS, Feneley MP, Hickey MS, Muhlbaier LH, Wechsler AS, Floyd RD, et al. A clinical comparison of mitral valve repair versus valve replacement in ischemic mitral regurgitation. J Thorac Cardiovasc Surg. 1988;95:165-77.

13. Tavakoli R, Weber A, Vogt P, Brunner HP, Pretre R, Turina M. Surgical management of acute mitral valve regurgitation due to post-infarction papillary muscle rupture. J Heart Valve Dis. 2002;11:20-5, discussion 6.

14. Bouma W, Wijdh-den Hamer IJ, Koene BM, Kuijpers M, Natour E, Erasmus ME, et al. Long-term survival after mitral valve surgery for post-myocardial infarction papillary muscle rupture. $J$ Cardiothorac Surg. 2015;10:11.

15. Russo A, Suri RM, Grigioni F, Roger VL, Oh JK, Mahoney DW, et al. Clinical outcome after surgical correction of mitral regurgitation due to papillary muscle rupture. Circulation. 2008;118:1528-34.

16. Morokuma H, Katayama Y, Koga S, Ogura Y. Anterior and posterior papillary muscle rupture after myocardial infarction. Asian Cardiovasc Thorac Ann. 2014;22:728-30. 